\title{
OLIVE PASTE CONSISTENCY AS A CONTROL PARAMETER FOR OIL EXTRACTION: A PRELIMINARY APPROACH
}

\author{
Piernicola Masella, Alessandro Parenti, Paolo Spugnoli
}

\section{Introduction}

Modern extra virgin olive oil processing plants usually operated in continuous mode [6].

The process consists in three most important steps: crushing of olive fruits that gives out an olive paste; continuous mixing (malaxation) of olive paste; oil extraction by means of paste centrifugation (horizontal decanter). Process optimization, in terms of maximal extraction yield, requires an understanding of how olive paste components are transformed by technological treatments (type, size and spatial distribution of particles, particles interactions) and how flow behavior of olive paste can be related to processing conditions [7]. Olive paste is a coarse solid-liquid mixture consisting of two distinct liquid phases (oil and water) and an extremely heterogeneous solid phase. Flow behavior of this material, i.e. its rheological properties, were characterized like a time-independent shear-thinning (pseudoplastic) fluid with the presence of a yield stress and simulated by means of Herschel-Bulkley model [2-5]. It has been highlighted that process conditions affect the flow behavior of the paste, mainly the mixing step involve a decrements in the apparent viscosity and in the consistency index (Herschel-Bulkley model). Malaxation is a critical step of the process because it allows to reduce or removes the emulsion of olive paste caused by the crusher, favorite the cohesion of smaller oil droplets into larger easier to separate drop and aid the breakage of the unbroken cells containing oil; therefore it increase the oil extraction yield [6]. Operative conditions, i.e. temperature and time, adopted during mixing can strongly increase the extraction yield, but they are subject to constraints coming from product quality [6]. At present, malaxation control is based upon accumulated empirical results and the experience of the operators. There are evidences that this method rarely identifies the olive-paste state suitable

\section{Paper received 31.08.2007; accepted 21.10.2008}

Prof. Ing. Paolo Spugnoli, Professore Ordinario; Prof. AlessanDro Parenti, Professore Associato; Dott. Piernicola Masella, Dottore di Ricerca. for oil extraction. Therefore detecting a measurable parameter able to monitor the olive-paste state suitable for oil extraction would be really useful. The paper reports the results of an investigation aimed to verify the feasibility of using the olive paste consistency variation during malaxation as a parameter representing the right state for oil extraction.

\section{Materials and methods}

Experimental Procedure - Olive oil extraction usually involves processing of material with different chemical and physical characteristics, i.e. olives of different origin, variety and ripeness state. This latter parameter, as related to external factors such as the weather, greatly influence the drupe moisture content, i.e. one of the most important parameter for plant settings during the extraction process [6]. On the basis of this considerations a set of experiments have been carried out on olive pastes (Frantoio var.) with different humidity content, i.e. 46,53 and $63 \%$ so to simulate processing of material of different initial characteristics. At this purpose olive paste with humidity content of $46 \%$ (w/w) was added with appropriate water amount so to reach the above mentioned humidity levels. In this way information about the influence of drupe with initial different humidity on oil extractability were achieved. The humidity content was determined by drying samples of about $50 \mathrm{~g}$ for $8 \mathrm{~h}$ at $105^{\circ} \mathrm{C}$ (mechanical convection oven); then, the samples were cooled for $30 \mathrm{~min}$ in a desiccator and reweighed. Olives were processed into paste with a lab extrusion mill. Then, the paste samples (about $1 \mathrm{~kg}$ ) were malaxed for different time $(0,15,30$ and $45 \mathrm{~min})$ at the same temperature $\left(28^{\circ} \mathrm{C}\right)$ by means of a lab vertical mixer equipped with a water bath for temperature control. Each malaxation trial was performed in triplicate. After malaxation each paste sample was divided in two equal aliquots for extraction yield and paste consistency evaluation.

Extraction Yield - To evaluate the extraction yield an oil extractability index (EI) was determined. At this purpose, from each olive paste sample, two aliquots of about $40 \mathrm{~g}$ were withdrawn and centrifuged 
(Beckman mod. JA 21) separately in two different conditions. After centrifugation, the oil was decanted in a graduated tube to measure the volume obtained to calculate the oil yield, which was expressed as a percentage of the fresh weight. The first paste aliquot of each sample was centrifuged at $4192 \mathrm{x} g$ for 40min: the extracted oil, expressed as percentage of the initial paste weight, was assumed as maximum extraction yield. In fact, preliminary tests showed that continuing centrifugation for a longer time practically no more oil was extracted. The second paste aliquot was centrifuged at $670 \mathrm{x} g$ for $2 \mathrm{~min}$ : the separated oil, expressed as percentage of the initial paste weight, was assumed as relative extraction yield. Therefore, the extractability index was given by the ratio:

$$
E I(\%)=\frac{\text { relative extraction yield }(\% \mathrm{w})}{\text { maximum extraction yield }(\% \mathrm{w})}
$$

In this way we can define the EI as the percentage of the potential extractable oil by means of centrifugation, i.e. physically extracted. The above-described procedure was repeated in duplicate for all the olive paste samples.

Consistency evaluation - A rotational torque rheometer (Rheolab MC1, Paar-Physica GmbH, Germany) was used to evaluate olive paste consistency. The instrument was configured for use as a concentric cylinder rheometer but providing to replace the inner cylinder with a steel rotating vane (Fig. 1) purposely designed for this experiment $[1,7]$.

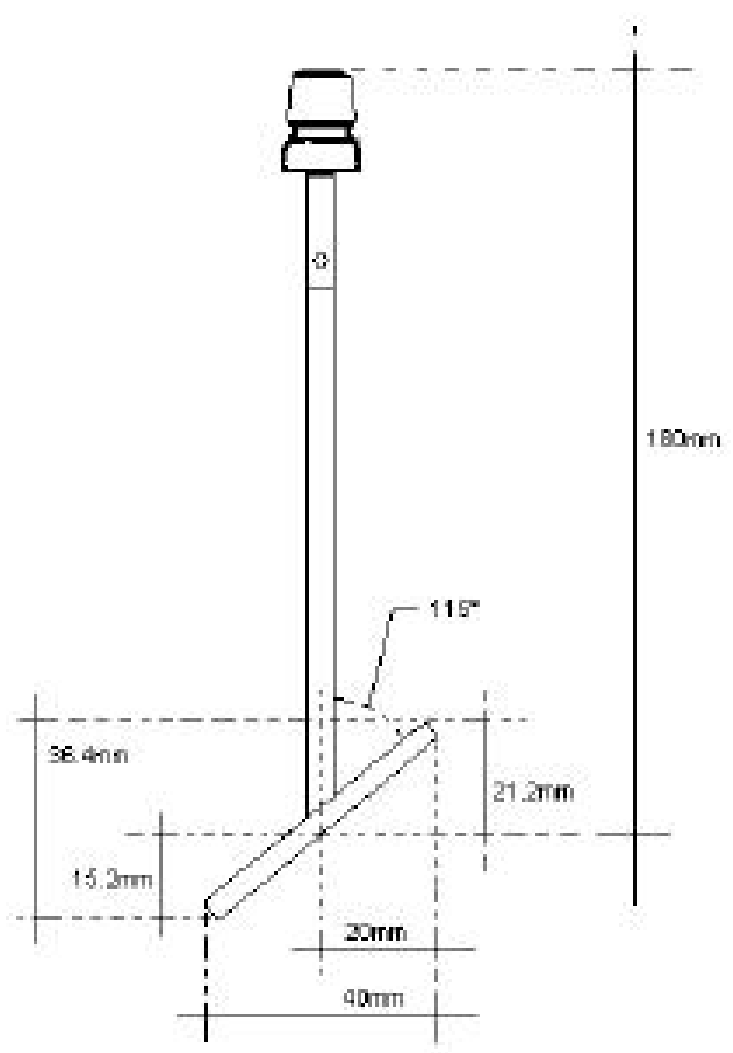

Fig. 1 - Geometric parameters of the vane measuring system used for paste consistency evaluation.
The outer cylinder, which corresponded to the measuring cup, had an internal total volume of $0.25 \mathrm{~L}$. The instrument was also equipped with a liquid temperature control system (TEZ180/MC1) enabling the user to precisely regulate the sample temperature. A Pt 100 resistance thermometer was used to measure the temperature either in the thermostatization fluid (water), in the measuring cup wall or directly in the sample. As the torque is directly linked to the product rheological properties, paste consistency was evaluated by directly reading the absolute torque required for the vane rotation, as a function of the increasing rotational speed (linear acquisition in a range of 1$60 \mathrm{rpm})$. Each measurement was repeated in duplicate.

\section{Results}

A typical result of olive paste consistency measurement is presented in Fig. 2. The graph reports the evolution of the torque as a function of the increasing rotation speed of the vane, in correspondence of four different malaxation time (paste humidity 53\%).

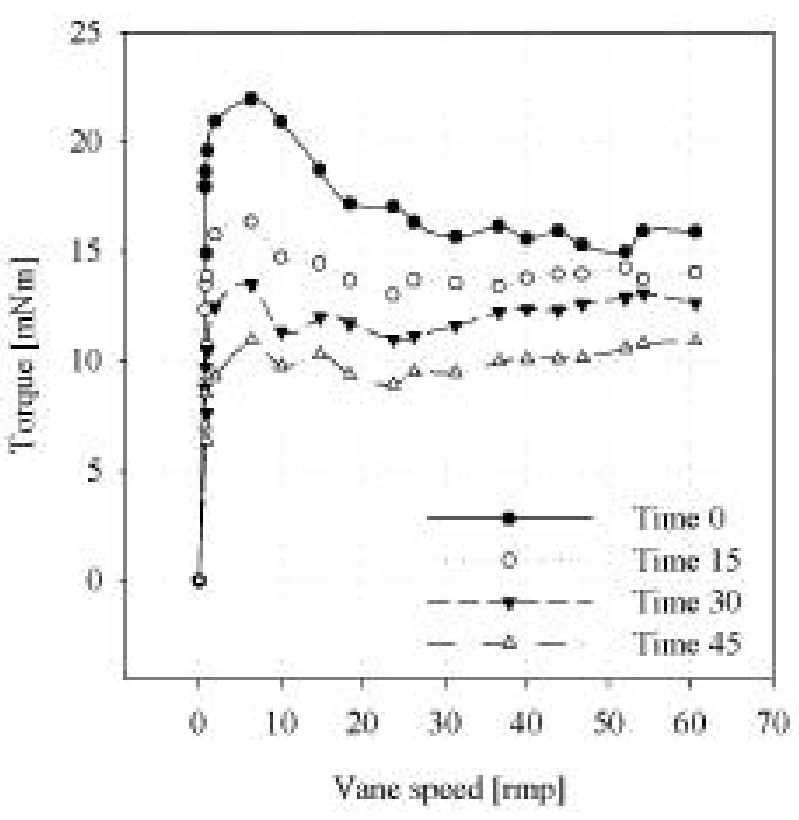

Fig. 2 - Example of olive paste consistency measurement.

All the paste samples showed essentially the same flow behavior as a function of the increasing rotational speed of the vane, i.e. an initial quasi-linear increment of the required torque until a maximum value (peak torque). Then, the required torque decreases, attaining lower steady-state values (speed $>30 \mathrm{rpm}$ ). A similar pattern was recorded for all the paste samples malaxated during the experiment and no substantial differences were found as a function of the different humidity content. Therefore the average value of the aforementioned torque steady values (eight points, vane speed $>30$ rpm), namely pastes consistency, was used in the fol- 
lowing to compare the different samples. The graph also clearly showed the effect of malaxation time which determined a large decrement of the required torque, therefore of the paste consistency. Significance of these differences was reported in Table 1 for the three levels of paste humidity content.

\begin{tabular}{|ccccc|}
\hline Paste & \multicolumn{4}{c|}{ Required torque $\mathbf{m N m}^{\mathrm{s}}$} \\
\cline { 2 - 5 } $\begin{array}{c}\text { Humidity } \\
\%\end{array}$ & time $=0$ & time $=15$ & time $=30$ & time $=45$ \\
\hline 47 & $19.3^{\mathrm{a}, \mathrm{x}}$ & $15.6^{\mathrm{b}, \mathrm{x}}$ & $14.5^{\mathrm{b}, \mathrm{x}}$ & $10.2^{\mathrm{c}, \mathrm{x}}$ \\
53 & $15.7^{\mathrm{a}, \mathrm{y}}$ & $13.8^{\mathrm{b}, \mathrm{y}}$ & $12.5^{\mathrm{c}, \mathrm{y}}$ & $10.1^{\mathrm{d}, \mathrm{x}}$ \\
63 & $12.8^{\mathrm{a}, \mathrm{z}}$ & $11.6^{\mathrm{ab}, \mathrm{z}}$ & $10.7^{\mathrm{bc}, \mathrm{z}}$ & $9.4^{\mathrm{c}, \mathrm{x}}$ \\
\hline
\end{tabular}

TABLE 1 - Variation of the required torque for vane rotation after different malaxation time and in correspondence of three level of paste humidity. ${ }^{\varsigma}$ Data are means of three independent replicates; within each row significant differences between means are shown by different letters from "a" to "d" (ANOVA, LSD test, $\mathrm{p}$ at 0.05 ); within each column significant differences between means are shown by different letters from "x" to " $z$ " (ANOVA, LSD test, p at 0.05).

As reported in the table, malaxation time strongly affect the paste consistency in all the paste samples. On the other hand it is also evident that paste consistency before malaxation was strictly related to the humidity content as stated by the extent of the differences in the required torque at time 0 . Further, these last differences showed a marked decrease during malaxation and at time $45 \mathrm{~min}$ no significant differences were found among the three humidity level. Therefore, it seems that after $45 \mathrm{~min}$ a consistency threshold value has been reached (torque about $10 \mathrm{mNm}$ ) over which further decrements were not detectable.

Similar consideration can be extended only partially

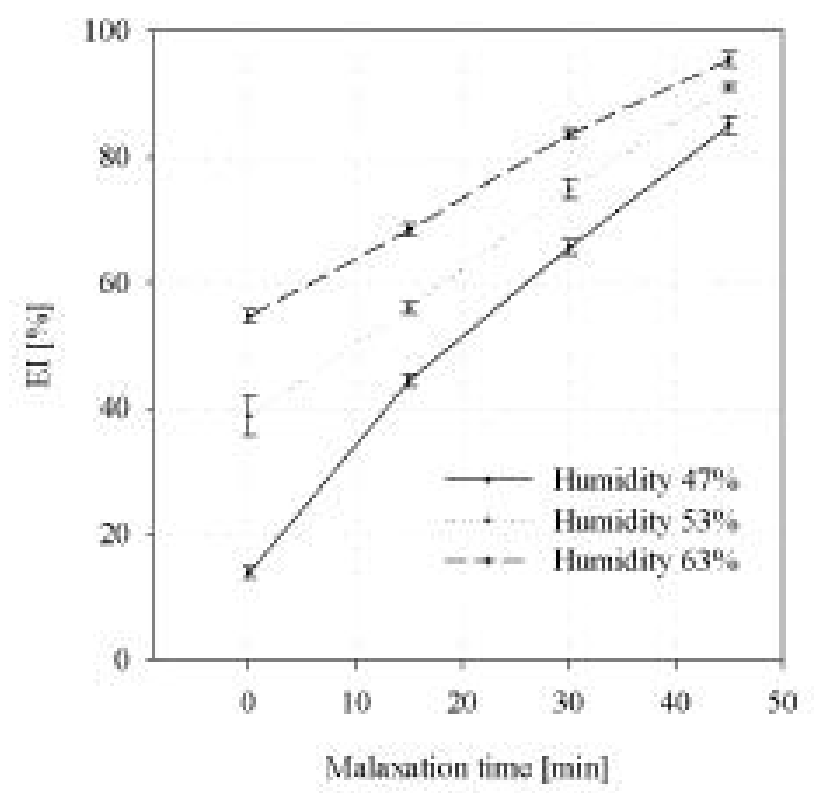

Fig. 3 - EI as a function of malaxation time; error bars correspond to standard deviations of three independent replicates. at the EI. As shown in Fig.3 all the paste samples showed a large increments of EI as a function of malaxation time. Nevertheless the paste with high humidity content showed all the time significantly much higher EI than those with lower humidity. Increasing time and water content have therefore a similar effect on the oil extractability and, differently with those observed for the paste consistency, significant differences were found after 45 min malaxation time as a function of the different humidity content. However, the differences in the EI as related to the different paste humidity content showed the tendency to decrease during malaxation (from about 20\% to about 5\%); in fact the paste with the lower humidity was more affected by malaxation time as compared to the other two samples showing an increment of about $70 \%$ (about 50 and $40 \%$ for paste humidity content of 53 and $63 \%$ respectively). Despite this dissimilar behavior of paste consistency and EI as a function of malaxation time, when the measure (torque) of paste consistency was plotted against the EI, a straight line is obtained (Fig. 4).

Descriptive parameters of the linear regression showed in Fig. 4 along with its statistical analysis are presented in Table 2.

\begin{tabular}{|lccccc|}
\hline Effect & Coeff. & SE & $\mathbf{t}$ & $\mathbf{p}$ & $\mathbf{r}^{2}$ \\
\hline intercept & 164.2 & 6.13 & 26.77 & 0.00 & \multirow{2}{*}{0.89} \\
slope & -7.66 & 0.46 & -16.67 & 0.00 & \\
\hline \multicolumn{7}{c|}{ Analysis of variance } \\
\hline Source & SS & df & MS & F-r & p \\
\hline $\begin{array}{l}\text { regression } \\
\text { residual }\end{array}$ & 17048.8 & 1 & 17048.8 & 277.9 & 0.00 \\
\hline
\end{tabular}

TABLE 2 - Analysis and parameters of linear regression of EI on torque (paste consistency). $\mathrm{SE}=$ standard error; $\mathrm{SS}=$ sum of squares; MS=mean squares; F-r= F ratio.

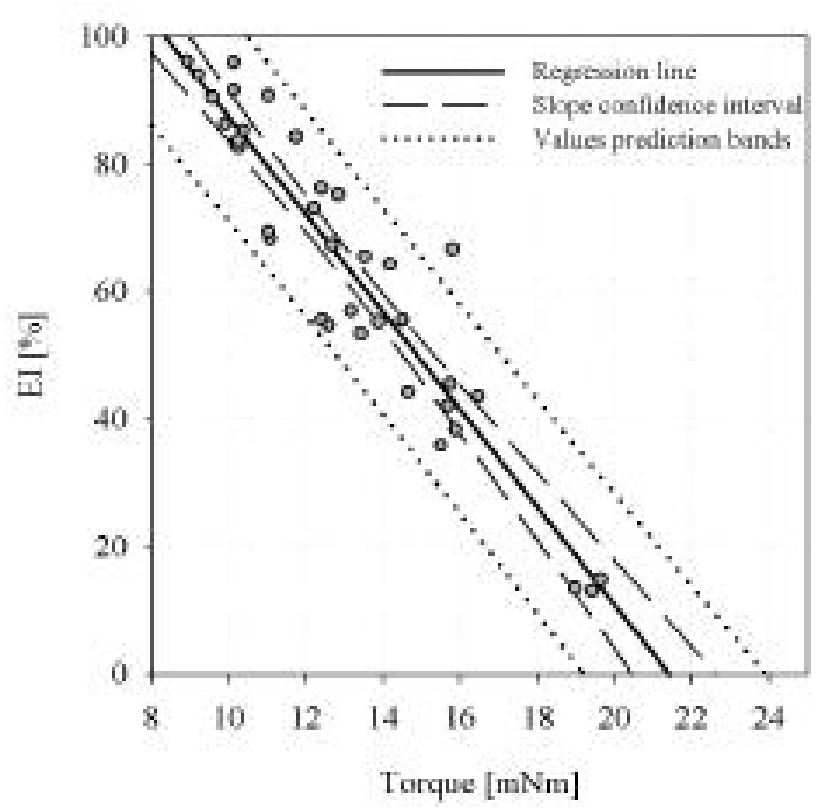

Fig. 4 - Linear regression of EI on torque. 
As stated by the statistical analysis a good linear regression subsist between EI and torque $(p<0.01)$ indicating that a large proportion of EI variance, about $90 \%$ (regression coefficient 0.89 ), can be explained in terms of paste consistency variation during malaxation. Although the regression showed a high slope value, i.e. high rate of EI units (about 7.6) per unit of torque, the goodness of the closeness of the linear relationship between the two variables indicate the feasibility to extrapolate from this observation to predict the right state of the paste for oil extraction.

\section{Conclusion}

From the results of this preliminary investigation it seems that a good relationship exists between oil extractability and paste consistency during the malaxation phase. This parameter therefore could be used as indicator of the right state of the olive paste for oil extraction. If the results will be confirmed in further investigations, and specifically at industrial plants scale, they could allow a consistent improvement in the olive-oil extraction process. As reported in the introduction, at the moment malaxation, despite its importance on oil extraction and quality, is performed practically without any control of the state of the paste. The conventional malaxation strategy adopted in olive oil processing plants consists of mixing a batch of olive paste with a constant-speed mixer at a more or less specified temperature for a more or less specified length of time. The availability of a measurable parameter representing the paste state suitable for oil extraction could allow to implement a "closed-loop" control system to optimize the process, that is: to detect the right moment for oil extraction related to the olive batches and malaxation temperature.

\section{References}

[1] Barnes H.A., NGuYen Q.D., Rotating vane rheometry a review, Non-Newtonian Fluid Mech. (2001) 98, 1-14.

[2] Catalano P., Montel G.L., Leone A., Tamborrino A., Influenza dei parametri di lavorazione sulle caratteristiche reologiche delle paste di olive snocciolate. Riduzione del costo di produzione, miglioramento della qualità e tutela dell'ambiente nella filiera, Progetto POM B02, Sciacca (AG) (2001) 1, 111-125.
[3] Di Renzo G.C., Colelli G., Flow behavior of olive paste, App Eng. Agric. (1997) 13, 751-755.

[4] Di Renzo G.C., Colelli G., Influenza dei parametri di lavorazione sulle caratteristiche reologiche delle paste di olive, Rivista di Ingegneria Agraria (1995) Quaderno n.17, 320-326.

[5] Formato A., Faugno S., Romano R., Paolillo G., Caratterizzazione meccanica delle paste di oliva, Convegno AIIA2005 (Catania, 27-30 giugno 2005), Codice lavoro: 7023.

[6] Harwood H., Aparicio R., Handbook of Olive oil, 5 ed. (2000), Aspen Publishers, Gaithersburg, MD (USA).

[7] STEFFE J.F., Rheological methods in food process engineering, 2a ed. (1996), Freeman Press (USA).

\section{SUMMARY}

Malaxation is a critical step of olive oil extraction process as it can largely affect the extraction yield along with product quality. At present, malaxation control is based upon accumulated empirical results and the experience of the operators. There are evidences that this method rarely point out the olive-paste state suitable for oil extraction. The paper reports the results of a laboratory investigation aimed to verify the feasibility of using the olive paste consistency variation during malaxation as a parameter representing the right state for oil extraction. Olive pastes consistency evaluation was performed with a rotational rheometer. Oil extraction yield was evaluated by means of an extractability index (EI) determined by laboratory centrifugation of the paste. The effect of different malaxation time and pastes humidity was investigated. Both malaxation time and humidity increments involve significant decrements of paste consistency and a simultaneous increase of EI. A good linear correlation among paste consistency and EI has been found, i.e. a large proportion of EI variance, about $90 \%$, can be explained in terms of paste consistency variation during malaxation. The availability of a measurable parameter representing the paste state suitable for oil extraction could allow to implement a "closed-loop" control system for extraction yield maximization.

\section{Key words:}

extraction yield, olive paste consistency, process control. 